\title{
CONGRUENCES CONTAINED IN AN EQUIVALENCE ON A SEMIGROUP
}

\author{
P. R. JONES \\ (Received 14 November, 1978; revised 8 March 1979) \\ Communicated by T. E. Hall
}

\begin{abstract}
The main theorem of this paper shows that the lattice of congruences contained in some equivalence $\pi$ on a semigroup $S$ can be decomposed into a subdirect product of sublattices of the congruence lattices on the 'principal $\pi$-factors' of $S$-the semigroups formed by adjoining zeroes to the $\pi$-classes-whenever these are well-defined. The theorem is then applied to various equivalences and elasses of semigroups to give some new results and alternative proofs of known ones.
\end{abstract}

1980 Mathematics subject classification (Amer. Math. Soc.) : primary 20 M 10; secondary 08 A 30.

\section{Introduction}

If $S$ is a semigroup and $\pi$ an equivalence on $S$, denote by $\Lambda^{\pi}(S)$ the complete lattice of congruences on $S$ contained in $\pi$ (and by $\Lambda(S)$ the lattice of all congruences on $S$ ). For each $\pi$-class of $S$ there is an associated groupoid formed by adjoining a zero to the class. If the groupoid is associative, we call this a principal $\pi$-factor of $S$. If every such groupoid is associative we say $\pi$ has principal $\pi$-factors on $S$. The Decomposition Theorem (see Section 2) shows that $\Lambda^{\pi}(S)$ is isomorphic with a subdirect product of sublattices of the congruence lattices of the principal $\pi$-factors, when they exist.

In Section 3, it is shown that every congruence $\pi$ has principal $\pi$-factors; so do $\mathscr{H}$ and $\mathscr{J}$ and in important cases $\mathscr{L}$ and $\mathscr{R}$. These are considered in Section 5. For example if $S$ is an inverse semigroup, $\Lambda^{\mathscr{f}}(S)$ is a subdirect product of ideals of the lattices of 0 -restricted congruences on its principal ( $\mathscr{J}$ ) factors. (If $S$ is completely semisimple it follows that every congruence contained in $\mathscr{J}$ is in fact contained in $\mathscr{H}$.) A theorem of Clifford and Preston (1967) on the structure on $\Lambda^{*}(S)$ is generalized to arbitrary semigroups, yielding an alternative (albeit longer) proof of Lallement's result on the modularity of $\Lambda^{*}(S)$. Semilattice decompositions are also 
considered-if $\tau$ is a semilattice congruence on $S$ with $\tau$-classes $S_{i}, i \in S / \tau$, then whenever $S$ is either inverse or a strong semilattice of the $S_{i}$ 's (but not generally) $\Lambda^{\tau}(S)$ is a subdirect product of the lattices $\Lambda\left(S_{i}\right), i \in S / \tau$.

In section 6, analogous results for left congruences are considered and a result of the author (1978a) on the semimodularity of the lattice of full inverse subsemigroups of an inverse semigroup is generalized.

Spitznagel (1973a, b), Eberhart and Williams (1978) and others have shown how information on $\Lambda^{\pi}(S)$ and $\Lambda(S / \pi)$ (for $\pi$ a congruence) may in certain cases be combined to give information on $\Lambda(S)$ itself. Their actual approaches differ from ours (although subdirect decomposition is implicit in their work).

\section{The Decomposition Theorem}

If $S$ is a semigroup denote by $\Lambda(S)$ (or just $\Lambda$ ) its lattice of congruences and by $\Lambda_{l}(S)$ $\left[\Lambda_{r}(S)\right]$ its lattice of left [right] congruences. Because of the obvious duality between $\Lambda_{l}$ and $\Lambda_{r}$ we shall in fact refer to $\Lambda_{l}$. The symbols $\xi$ and $\omega$ will denote the trivial and universal congruences, respectively, on $S$. If $S$ has a zero 0 then 0 -restricted left congruences of $S$ (those for which $\{0\}$ is a congruence class) form an ideal of $\Lambda(S)$ $\left[\Lambda_{n}(S)\right]$. Clearly if $S \backslash 0$ is a subsemigroup of $S$ then the lattice of 0 -restricted congruences on $S$ is isomorphic with $\Lambda(S \backslash 0)$.

For all undefined terms and notation the reader is referred to Howie (1976) or to Clifford and Preston (1961, 1967), for semigroups, and to Birkhoff (1967) for lattice theory.

Let $\pi$ be an equivalence on $S$. Put $\Lambda^{\pi}(S)=\{\rho \in \Lambda(S): \rho \subseteq \pi\}$. Then $\Lambda^{\pi}$ is a complete ideal of $\Lambda$ (Howie (1976), p. 27). Define $\Lambda_{l}^{\pi}(S)=\left\{\rho \in \Lambda_{l}(S): \rho \subseteq \pi\right\}$, similarly.

Suppose the $\pi$-classes of $S$ are $\left\{S_{i}: i \in S / \pi\right\}$. For each $i \in S / \pi$, let $P_{i}=S_{i}^{0}$ be the groupoid $S_{i} \cup\{0\}$ (that is if $S_{i}$ contains a zero, a new zero is to be adjoined) where all products not falling in $S_{i}$ are 0 . In general $P_{i}$ need not be a semigroup.

Definition. For each $i \in S / \pi, P_{i}$ is a principal $\pi$-factor of $S$ if it is a semigroup. If every $P_{i}$ is a principal $\pi$-factor, then $\pi$ has principal factors on $S$.

In Section 3 we will consider which equivalences have principal factors. For now we just comment that the principal $\mathscr{J}$-factors of $S$ are essentially the usual principal factors of $S$.

THEOREM 2.1 (Decomposition Theorem). Let $S$ be a semigroup and $\pi$ an equivalence on $S$ having principal factors $P_{i}, i \in S / \pi$. Then $\Lambda^{\pi}\left[\Lambda_{l}^{\pi}\right]$ is a subdirect product of sublattices of the lattices $\Lambda\left(P_{i}\right)\left[\Lambda_{i}\left(P_{i}\right)\right], i \in S / \pi$. 
ProOF. If $\rho$ is any equivalence on $S$ such that $\rho \subseteq \pi$, let $\rho_{i}=\rho \mid S_{i} \cup\{(0,0)\}$ for each $i$ (where $\rho \mid S_{i}=\rho \cap\left(S_{i} \times S_{i}\right)$ and $S_{i}$ is the $\pi$-class associated with $\left.P_{i}\right)$. Clearly $\rho_{i}$ is a 0 restricted equivalence on $P_{i}$. Moreover $\rho \bigcup\left\{\rho \mid S_{i}: i \in S / \pi\right\}$.

We now show that if $\rho \in \Lambda_{l}^{\pi}$ then $\rho_{i}$ is a left congruence of $P_{i}$. Let $(a, b) \in \rho_{i}, a \neq b$, and let $s \in P_{i}$. If $s=0$, then $s a=s b=0$. Otherwise $s \in S_{i}$ and $a, b \in S_{i}$ (since $\rho_{i}$ is 0 restricted and $a \neq b)$ so $(a, b) \in \rho$, whence $(s a, s b) \in \rho$. But $\rho \subseteq \pi$, so either $s a=s b=0$ in $P_{i}$ or $s a, s b \in S_{i}$. In either case $(s a, s b) \in \rho_{i}$, as required.

The map $\rho \mapsto \rho_{i}$ of $\Lambda_{i}^{\pi}(S)$ into $\Lambda_{l}\left(P_{i}\right)$ is therefore well-defined for every $i$. Moreover, each map is intersection-preserving. Now let $\rho, \sigma \in \Lambda_{l}^{\pi}(S)$ and $i \in S / \pi$. Since $(\rho \vee \sigma)_{i}$ contains $\rho_{i}$ and $\sigma_{i}$, we have $\rho_{i} \vee \sigma_{i} \subseteq(\rho \vee \sigma)_{i}$ in $\Lambda_{l}\left(P_{i}\right)$. Conversely, let $(a, b) \in(\rho \vee \sigma)_{i}$, $a \neq b$, so that $(a, b) \in \rho \vee \sigma$, in $\Lambda_{l}(S)$, as above. Then there is a sequence $a=x_{0}, x_{1}, \ldots, x_{n}=b$ of elements of $S$ such that $\left(x_{i-1}, x_{i}\right) \in \rho \cup \sigma, 1 \leqslant i \leqslant n$. But $\rho \cup \sigma \subseteq \pi$ and $S_{i}$ is a $\pi$-class containing $x_{0}$, so each $x_{i} \in S_{i}$, that is $\left(x_{i-1}, x_{i}\right) \in \rho_{i} \cup \sigma_{i}$, $1 \leqslant i \leqslant n$. Therefore $(a, b) \in \rho_{i} \vee \sigma_{i}$ so that $(\rho \vee \sigma)_{i} \subseteq \rho_{i} \vee \sigma_{i}$ in $\Lambda_{l}\left(P_{i}\right)$.

Hence each map $\rho \mapsto \rho_{i}$ is a lattice morphism of $\Lambda_{l}^{\pi}(S)$ into $\Lambda_{l}\left(P_{i}\right)$.

Now if $\rho, \sigma \in \Lambda_{l}^{\pi}$ and $\rho_{i}=\sigma_{i}$ for all $i \in S / \pi$, then $\rho_{i}\left|S_{i}=\sigma_{i}\right| S_{i}$ for all $i \in S / \pi$, whence $\rho=\sigma$. The result for $\Lambda_{i}^{\pi}$ now follows from (for example) (11.1) of Crawley and Dilworth (1973).

Clearly when $\rho \in \Lambda^{\pi}(S)$, each $\rho_{i}$ is a congruence on $P_{i}$ and the result for $\Lambda^{\pi}$ follows similarly. //

From the proof of the theorem it is clear that each subdirect factor of $\Lambda^{\pi}\left[\Lambda_{l}^{\pi}\right]$ consists of those 0-restricted [left] congruences on the corresponding principal $\pi$ factor which are induced by some [left] congruence contained in $\pi$ (in the sense of the theorem). In the following two situations, which will occur frequently in the sequel, we can be more explicit. (Although we shall only state results concerning $\Lambda^{\pi}$, the analogues for $\Lambda_{l}^{\pi}$ are obvious).

Corollary 2.2. Let $\pi$ be an equivalence on $S$, with $\pi$-classes $S_{i}, i \in S / \pi$, and suppose each of these classes is a subsemigroup of $S$. Then $\Lambda^{\pi}$ is a subdirect product of sublattices of the lattices $\Lambda\left(S_{i}\right), i \in S / \pi$. Each subdirect factor consists of those congruences on the corresponding $\pi$-class which are the restrictions of congruences on $S$ contained in $\pi$.

Proof. Since each $S_{i}$ is a subsemigroup of the associated principal factor $P_{i}$ (clearly well-defined), this follows from the statements in the first paragraph of this section. //

COROLLARY 2.3. Let $\pi$ be an equivalence having principal factors on $S$ and suppose that on each principal $\pi$-factor of $S$ every 0 -restricted congruence is induced by some congruence on $S$. Then

(i) the subdirect factors of $\Lambda^{\pi}$ are ideals of the lattices of 0 -restricted congruences on the principal $\pi$-factors; 
(ii) if, further, $\pi$ is a congruence, then the subdirect factors are the lattices themselves.

NoTE. It is not assumed that the congruences are induced by congruences contained in $\pi$.

PROOF. Suppose $\rho$ is a 0 -restricted congruence on the principal $\pi$-factor $P$ which is induced by a congruence $\rho^{\prime}$, say, in $\Lambda^{\pi}(S)$. Let $\sigma \in \Lambda(P), \sigma \subseteq \rho$. Then $\sigma$ is 0-restricted and is induced by $\sigma^{\prime}$, say, $\sigma^{\prime} \in \Lambda(S)$. But then $\sigma$ is also induced by $\sigma^{\prime} \cap \rho^{\prime}$, in $\Lambda^{\star}(S)$, and (i) follows from the comments after the theorem.

If $\pi$ is a congruence then $\pi$ itself induces the congruence $(P \backslash 0) \times(P \backslash 0) \cup\{(0,0)\}$ on $P$ and this is the maximum 0 -restricted congruence on $P$. Hence the ideal in (i) is now the entire lattice of 0 -restricted congruences on $P$. //

This corollary has an obvious specialization to the situation of Corollary 2.2 .

To avoid repetition of details in the sequel we will state here a lemma on the recognition of those congruences on principal factors which are induced by congruences of $S$.

LEMMA 2.4. Let $\pi$ be an equivalence having principal factors on $S$ and let $A$ be $a \pi$ class with associated principal $\pi$-factor $P$. Let $\sigma$ be a 0 -restricted congruence on $P$. Then $\sigma$ is induced by some congruence on $S$ iffor all $(a, b) \in \sigma$ such that $a \neq b$, and for all $s, t \in S^{1}$ such that $s a t \in A$, then $s b t \in A$ and $(s a t, s b t) \in \sigma$.

Proof. Let $\sigma^{*}$ be the congruence on $S$ generated by the equivalence $\sigma \cup t_{S}$. Clearly $\sigma\left|A \subseteq \sigma^{*}\right| A$. To prove the reverse inclusion suppose $(x, y) \in \sigma^{*} \mid A$. Then $y$ is obtained from $x$ by a sequence of elementary $\sigma$-transitions $x=x_{0} \rightarrow x_{1} \rightarrow \ldots \rightarrow x_{n}=y$, where the $x_{i}$ may be assumed distinct. By hypothesis, since $x_{0} \in A$ then $x_{1} \in A$ and $\left(x_{0}, x_{1}\right) \in \sigma$. By repeating this we eventually obtain $(x, y) \in \sigma$. Hence $\sigma\left|A=\sigma^{*}\right| A$, as required. //

\section{Principal $\pi$-factors}

In this section many familiar equivalences are shown to have principal factors on any semigroup. Clearly any equivalence all of whose classes are subsemigroups has principal factors.

To avoid possible confusion we will, for the next lemma only, use the symbol $*$ to denote the operation on the groupoid $A^{0}, A \subseteq S$ (a semigroup) defined by

$$
a * b= \begin{cases}a b & \text { if } a, b, a b \in A, \\ 0 & \text { otherwise. }\end{cases}
$$


LEMMA 3.1. If $S$ is a semigroup and $A \subseteq S$, then $A^{0}$ is a semigroup if and only if whenever $a, b, c$ and $a b c \in A$, then $a b \in A$ if and only if $b c \in A$.

Proof. If $a, b, c \in A$ then

$$
(a * b) * c= \begin{cases}(a b) c & \text { if } a b,(a b) c \in A, \\ 0 & \text { otherwise }\end{cases}
$$

and

$$
a *(b * c)= \begin{cases}a(b c) & \text { if } b c, a(b c) \in A, \\ 0 & \text { otherwise. }\end{cases}
$$

Since $(a b) c=a(b c)$ in $S$, then $(a * b) * c=a *(b * c)$ for all $a, b, c \in A$ if and only if $a b c$ and $a b$ belong to $A$ precisely when $a b c$ and $b c$ belong to $A$. //

Since the principal $\pi$-factors of an equivalance are formed from the equivalence classes in just this way, we have

COROLlary 3.2. The equivalence $\pi$ on $S$ has principal factors if

(i) $\pi$ is any congruence on $S$,

(ii) $\pi=\mathscr{J}$, or

(iii) $\pi=\mathscr{H}$.

Proof. Let $A$ be a $\pi$-class and suppose $a, b, c$ and $a b c \in A$, that is $a \pi b \pi c \pi a b c$.

(i) If $\pi$ is a congruence on $S$, then $a b \pi b^{2} \pi b c$, so $a b \in A$ if and only if $b c \in A$.

(ii) If $\pi=\mathscr{J}$, then $A=J_{a b c} \leqslant J_{a b} \leqslant J_{a}=A$, that is $a b \in A$. Similarly $b c \in A$.

(iii) If $\pi=\mathscr{H}$ then by Green's Theorem (see Howie (1976), Theorem II2.5) either $A^{2} \cap A$ is empty or $A$ is a group. In either case the result is clear. //

REMARKS. 1. The principal $\mathscr{J}$-factors of $S$ are just the principal factors in the usual terminology (except that if $S$ has a minimum $\mathscr{J}$-class, the zero is more usually omitted from that factor).

2. The principal $\mathscr{H}$-factors of $S$ are either groups with zero or zero semigroups.

We will show that $\mathscr{L}$ and $\mathscr{R}$ need not have principal factors on arbitrary semigroups. However, they do on completely semisimple semigroups. (Recall from Clifford and Preston (1967), Chapter 6, that $S$ is completely semisimple if each principal $\mathscr{J}$-factor is completely 0 -simple, in which case $S$ is regular and $\mathscr{D}=\mathscr{J}$. Every finite regular semigroup is completely semisimple.)

PROPOSITION 3.3. If $S$ is completely semisimple then $\mathscr{L}$ and $\mathscr{R}$ have principal factors. 
Proof. By duality it is sufficient to consider $\mathscr{R}$.

Let $A$ be an $\mathscr{R}$-class of $S$ and suppose $a, b, c$ and $a b c \in A$. We may consider $A$ as a subset of its associated completely 0 -simple principal $\mathscr{J}$-factor $P$. Now $a b c \neq 0$ in $P$, whence $b c \neq 0$ also. By Theorem 2.52(ii) of Clifford and Preston (1961), $b c \in R_{b} \cap L_{c} \subseteq A$. Similarly $a b \neq 0$ and $a b \in A$. //

For arbitrary semigroups this need not be so. For example let $B$ be the bicyclic semigroup, represented as $\mathbf{N} \times \mathbf{X}$ ( $\mathbf{N}$ is the set of non-negative integers) with product

$$
(m, n)(p, q)=(m-n+\max (n, p), q-p+\max (n, p)),
$$

and consider the $\mathscr{R}$-class $R=R_{(1,1)}=\{1\} \times \mathbf{N}$. Let $a=(1,2), b=(1,0)$ and $c=(1,3)$. Now $a b=(1,1) \in R$ and $a b c=(1,3) \in R$ but $b c=(2,3) \notin R$. By the lemma $\rightarrow$ does not have principal factors in $B$.

In fact since any regular semigroup which is not completely semisimple contains a copy of $B$, on which $\mathscr{R}_{B}=\mathscr{R}_{S} \cap(B \times B)$ (Hall (1972)), it follows that on a regular semigroup $S, \mathscr{R}$ (and dually $\mathscr{L}$ ) has principal factors if and only if $S$ is completely semisimple. (On (irregular) free semigroups, for instance, $\mathscr{R}$ is, however, trivial and hence has principal factors).

The position with $\mathscr{D}$ is unclear.

\section{Semimodularity}

Before applying the results of the previous two sections a short digression into lattice theory is required.

Following Crawley and Dilworth (1973) we will call a lattice $L$ semimodular if $a \succ a \wedge b$ implies $a \vee b \succ b$ for all $a, b \in L$ (Here $x>y$ means $x$ covers $y$, that is $x>y$ and for no $z$ in $L$ is $x>z>y$.) Following F. Maeda and S. Maeda (1970), we call $L M$-symmetric if the modularity relation $M$ on $L$ is symmetric, where

$$
a M b \text { if for all } x \in[a \wedge b, b], b \wedge(x \vee a)=x .
$$

We now summarize the properties of $M$-symmetric lattices. For proofs, or references to proofs, see Jones (1978a), where extensive use is made of the concept.

ReSULT 4.1. (i) Modularity $\Rightarrow M$-symmetry $\Rightarrow$ semimodularity.

(ii) $M$-symmetry is preserved by ideals, but not by sublattices in general, nor by lattice morphisms.

(iii) $M$-symmetry is preserved by subdirect products.

(iv) For any set $I$, the lattice $\Pi(I)$ of equivalences on $I$ is $M$-symmetric.

Clearly (iii) is relevant in our situation. In fact (iii) is the only part of the result which is not true for semimodularity also. (This may explain the reason for preferring 
the stronger concept here. Actually, for lattices of finite length the two properties are equivalent).

It was shown by Lallement (1967) that $\Lambda(S)$ is semimodular if $S$ is completely 0 simple. From his description of the congruences on $S$ it is readily seen that if we represent $S$ as $\mathscr{M}^{0}(G ; I, \Sigma ; P)$, then the lattice of 0 -restricted (that is non-universal, in this case) congruences on $S$ is a subdirect product of $\mathcal{N}(G)$ and ideals of $\Pi(I)$ and $\Pi(\Sigma)$, where $\mathcal{N}(G)$ is the (modular) lattice of normal subgroups of $G$. Hence

Proposition 4.2. If $S$ is completely 0 -simple, the lattice of 0-restricted congruences is $M$-symmetric (and hence so is $\Lambda(S)$ itself). In particular if $S$ is completely simple, $\Lambda(S)$ is M-symmetric. //

Johnston (1978) made a similar observation regarding the subdirect decomposition but incorrectly deduced semimodularity from it.

An alternative approach to decomposing congruence lattices has been pursued by Spitznagel (1973a,b) and Eberhart and Williams (1978), in which subdirect decompositions are implicit. We will not use their results but will mention results similar to our own. However, one result which is worth quoting, in modified form, is Theorem 1.3 of Eberhart and Williams (1978).

If $L$ is a lattice, $z \in L$, then $z$ is a neutral element of $L$ if (i) $z \wedge a=z \wedge b$ and $z \vee a=z \vee b$ together imply $a=b$, for any $a, b \in L$ and (ii) the maps $z \rightarrow z \wedge a$ and $z \rightarrow z \vee a, a \in L$, are endomorphisms of $L$.

Then (Birkhoff (1967), p. 69) if $z$ is neutral, $L$ is a subdirect product of the ideal $L \wedge z$ and the dual ideal $L \vee z$.

RESULT 4.3. If $z$ is a neutral element of the lattice $L$, then $L$ is semimodular if and only if both $L \wedge z$ and $L \vee z$ are. //

For $M$-symmetric lattices this is immediate from Result 4.1. (Note that Eberhart and Williams use a slightly different definition of 'semimodularity'.)

\section{Applications to congruences}

From the Decomposition Theorem it is immediate that if $\pi$ has principal factors each of whose lattices of congruences satisfies a given identity (for instance modularity or distributivity) then $\Lambda^{\pi}$ also satisfies that identity. Semigroups with modular congruence lattices are rather scarce, however, and we shall be more concerned with semimodularity (via $M$-symmetry). One application to modularity is to be found by considering $\mathscr{H}$, which by Corollary 3.2 has principal factors. 
It was shown by Lallement (1966) that $\Lambda^{\star}$ is modular for any semigroup, thus generalizing the special cases proved by Clifford and Preston (1967), Theorem 7.58, and Munn (1964) for inverse and regular semigroups respectively. Both Lallement and Munn proved modularity by showing the congruences contained in $\mathscr{H}$ commute. Clifford and Preston essentially proved that on an inverse semigroup $\Lambda^{*}$ is a subdirect product of sublattices of the lattices of normal subgroups of its maximal subgroups and it is this result which we may now easily generalize to arbitrary semigroups.

An arbitrary semigroup may have no subgroup whatsoever. However with any $\mathscr{H}$-class $H$ can be associated the Schutzenberger group $\Gamma(H)$ of $H$ : the group of all transformations of $H$ induced by inner right translations of $S^{\mathbf{1}}$. Then (Schutzenberger (1957)) $\Gamma(H) \cong \Gamma\left(H^{\prime}\right)$ if $H$ and $H^{\prime}$ are contained in the same $\mathscr{D}$-class of $S$. If that $\mathscr{D}$-class is regular then $\Gamma(H)$ is isomorphic with each of its maximal subgroups.

THEOREM 5.1. If $S$ is any semigroup, $\Lambda^{*}(S)$ is a subdirect product of sublattices of the lattices of normal subgroups of the Schutzenberger groups of $S$. Hence $\Lambda^{*}(S)$ is modular.

Proof. By the Decomposition Theorem, $\Lambda^{*}(S)$ is a subdirect product of the lattices of 0 -restricted congruences of the principal $\mathscr{H}$-factors of $S$.

Let $H$ be an $\mathscr{H}$-class of $S$. For each 0-restricted congruence (on the associated principal factor) which is induced by some congruence $\sigma^{\prime}$, say, of $S$, define an equivalence $E(\sigma)$ on $\Gamma(H)$ by

$$
E(\sigma)=\{(\lambda, \mu) \in \Gamma(H) \times \Gamma(H):(h \lambda, h \mu) \in \sigma \text { for all } h \in H\} .
$$

Let $(\lambda, \mu) \in E(\sigma)$ and let $\eta \in \Gamma(H), \eta=\rho_{s} \mid H$, say, where $s \in S^{1}$ and $x \rho_{s}=x s$ for all $x \in S$. Then for all $h$ in $H$

$$
(h(\lambda \eta), h(\mu \eta))=((h \lambda) s,(h \mu) s) \in \sigma^{\prime} \mid H=\sigma,
$$

and

$$
(h(\eta \lambda), h(\eta \mu))=((h \eta) \lambda,(h \eta) \mu) \in \sigma,
$$

since $h \eta \in H$. Thus $(\lambda \eta, \mu \eta) \in E(\sigma)$ and $(\eta \lambda, \eta \mu) \in E(\sigma)$. So $E(\sigma)$ is a congruence on $\Gamma(H)$. It is easily verified that the map $\sigma \rightarrow E(\sigma)$ is an embedding of the associated subdirect factor of $\Lambda^{*}$ into $\Lambda(\Gamma(H)$ ), that is into the lattice of normal subgroups of the Schutzenberger group of $H$. //

Turning now to $\mathscr{J}$, it is immediate from the Decomposition Theorem that $\Lambda^{f}(S)$ is a subdirect product of sublattices of the congruence lattices of its principal $(\mathscr{J}-)$ factors. We will consider inverse semigroups first. 
In a completely semisimple inverse semigroup (see Section 3 ) each principal factor is a Brandt semigroup and on such a semigroup every 0-restricted congruence separates idempotents (since if $e$ and $f$ are related idempotents either $e=f$ or $e f=0$ ). Hence it is immediate from the Decomposition Theorem (and the comments following it) that every congruence contained in $\mathscr{J}$ is idempotent-separating. Thus we have

Corollary 5.2. On a completely semisimple inverse semigroup every congruence contained in $\mathscr{J}$ is contained in $\mathscr{H}$, that is $\Lambda^{\mathscr{F}}=\Lambda^{*}$.

In general this need not, of course, be true even for inverse semigroups, as any simple inverse semigroup which is not a group has $\mathscr{J}=\omega$ and $\mathscr{H} \subset \mathscr{J}$.

To obtain deeper results for inverse semigroups it is necessary to further analyse the subdirect factors (along the lines of Section 2). The extra hypotheses in the next lemma will be used in the sequel.

LEMMA 5.3. Let $S$ be an inverse semigroup and let $\pi$ be either $\mathscr{J}$ or a congruence containing $\mathscr{F}$. If $P$ is a principal $\pi$-factor of $S$ then every 0 -restricted congruence on $P$ is induced by some congruence on $S$.

Proof. Before commencing the proof proper, we will show that if $a$, in $S$, is such that sat $\pi a$ for some $s, t \in S^{1}$, then $s a a^{-1} \pi a \pi a^{-1} a t$.

Now $s a t=\left(s a a^{-1}\right) a\left(a^{-1} a t\right)$, so $J_{s a t} \leqslant J_{s a a^{-1}} \leqslant J_{a}$. If $\pi=\mathscr{J}$ then clearly saa ${ }^{-1} \pi a$. If $\pi$ is a congruence containing $\mathscr{J}$, note that there exist idempotents $e$ of $J_{\text {sat }}, f, g$ of $J_{s a a^{-1}}$ and $h$ of $J_{a}$ such that $e \leqslant f$ and $g \leqslant h$ (by, for example, Exercise 3 of Clifford and Preston (1967), Chapter 8). Then since $\mathscr{J} \subseteq \pi$ we have $e \pi$ sat $\pi a \pi h$ and $f \pi g$, whence $e=e f \pi g h=g$, so that a $\pi \mathrm{sat} \pi s a a^{-1}$. Similarly $a^{-1}$ at $\pi a$ in either case.

To apply Lemma 2.4, let $A$ be the associated $\pi$-class of $S$, let $\sigma$ be a 0 -restricted congruence on $P$ and suppose $(a, b) \in \sigma, a \neq b$, and sat $\in A$ for some $s, t \in S^{1}$.

Then sat $\pi a$ so that $s a a^{-1}, a^{-1} a t \in A$ by the above. Therefore $s a t=\left(s a a^{-1}\right) a\left(a^{-1} a t\right) \sigma\left(s a a^{-1}\right) b\left(a^{-1} a t\right) \in A$ (since $\sigma$ is 0-restricted). Similarly $s a a^{-1} b b^{-1}, b^{-1} b a^{-1} a t \in A$ so

$$
\begin{aligned}
s a a^{-1} b a^{-1} a t & =\left(s a a^{-1} b b^{-1}\right) b\left(b^{-1} b a^{-1} a t\right) \sigma\left(s a a^{-1} b b^{-1}\right) a\left(b^{-1} b a^{-1} a t\right) \\
& =\left(s b b^{-1}\right) a\left(b^{-1} b t\right) \in A .
\end{aligned}
$$

Again $s b b^{-1}, b^{-1} b t \in A$ and so

$$
\left(s b b^{-1}\right) a\left(b^{-1} b t\right) \sigma\left(s b b^{-1}\right) b\left(b^{-1} b t\right)=s b t .
$$

So $s b t \in A$ and $(s a t, s b t) \in \sigma$. By Lemma 2.4 , every 0 -restricted congruence on $P$ is induced by some congruence on $S$. // 
THEOREM 5.4. If $S$ is an inverse semigroup then $\Lambda^{\prime}(S)$ is a subdirect product of ideals of the lattices of (0-restricted) congruences of its principal $(\mathscr{J}-)$ factors.

Proof. Apply Corollary 2.3 to the Decomposition Theorem, using the lemma.

Since $M$-symmetry is preserved by ideals, the theorem shows that if each principal factor of $S$ has an $M$-symmetric lattice of $\left(0\right.$-restricted) congruences then $\Lambda^{\prime}(S)$ is $M$ symmetric (whence semimodular). However, 0 -simple inverse semigroups need not in general have semimodular lattices of (0-restricted) congruences (see Scheiblich (1970)).

Returning to the extra hypotheses of Lemma 5.3, a congruence $\tau$ on $S$ containing $\mathscr{J}$ is just a semilattice congruence (Howie and Lallement (1966)) in which case each congruence class $S_{i}$ is an inverse subsemigroup of $S$ and $S$ is a semilattice of the subsemigroups $S_{i}, i \in S / \tau$. Thus, applying Corollaries 2.2 and 2.3 we have

THEOREM 5.5. If $S$ is a semilattice of inverse subsemigroups $S_{i}, i \in I$, with associated congruence $\tau$, then $\Lambda^{\mathrm{\tau}}(S)$ is a subdirect product of the lattices $\Lambda\left(S_{i}\right), i \in I$. //

This theorem can also be interpreted in the opposite direction: a necessary condition that $\Lambda^{\tau}(S)$, indeed $\Lambda(S)$ itself, satisfy some lattice identity is that the congruence lattices of the inverse semigroups in any semilattice decomposition (in particular that corresponding to the least semilattice congruence) also satisfy that identity.

We now consider semilattice decompositions of arbitrary semigroups. In general, the results are not as definitive as Theorem 5.5, even for regular or completely semisimple semigroups. If $S$ is a semilattice of subsemigroups $S_{i}, i \in I$, with associated congruence $\tau$, then $\Lambda^{\tau}(S)$ is of course a subdirect product of sublattices of the lattices $\Lambda\left(S_{i}\right), i \in I$ (by Corollary 2.2) but the subdirect factors need not even be ideals.

Example 5.6. Let $R=\{s, t\}$ and $K=\{a, b, c, d\}$ be right zero semigroups and let $S=R \cup K$, with $s x=t x=x$ for all $x$ in $K$ and $a s=a=c s, b s=b=d s, a t=c=c t$, $b t=d=d t$. It may be verified that $S$ is a band. Further $\sigma=\{(a, b),(b, a)\} \cup l_{K}$ is a congruence on $K$. But $(a t, b t)=(c, d) \notin \sigma$, so $\sigma$ is not induced by any congruence on $S$. Since $\omega_{K}$ is induced by the congruence $\mathscr{J}$ on $S$, the congruences on $K$ induced by congruences on $S$ contained in $\mathscr{J}$ do not form an ideal of $\Lambda(K)$.

If, however, $S$ is a strong semilattice of the $S_{i}$ 's we now show the analogue of Theorem 5.5 is true. (Recall (Petrich (1973)) that if $S$ is a semilattice $Y$ of semigroups $S_{i}, i \in Y$, then $S$ is a strong semilattice of the $S_{i}$ 's if there exist maps $\varphi_{i, j}: S_{i} \rightarrow S_{j}$ whenever $i \geqslant j$, satisfying

(i) $\varphi_{i, i}$ is the identity on $S_{i}$, 
(ii) $\varphi_{i, j} \varphi_{j, k}=\varphi_{i, k}, i \geqslant j \geqslant k$,

(iii) if $a \in S_{i}$ and $b \in S_{j}$ then $a b=\left(a \varphi_{i, i j}\right)\left(b \varphi_{j, i j}\right)$.

LEMMA 5.7. If $S$ is a strong semilattice of the semigroups $S_{i}, i \in Y$, with associated congruence $\tau$, then for each $i$, every congruence on $S_{i}$ is the restriction of some congruence on $S$.

Proof. Let $i \in Y$ and $\sigma \in \Lambda\left(S_{i}\right)$. Let $(a, b) \in \sigma, a \neq b$, and suppose sat $\in S_{i}$ for some $s, t \in S^{1}$. We may assume $s, t \neq 1$ (the proof being similar if $s=1$ or $t=1$ ). Suppose $s \in S_{j}$ and $t \in S_{k}, j, k \in Y$.

Now sat $=\left(s \varphi_{j, i j k}\right)\left(a \varphi_{i, i j k}\right)\left(t \varphi_{k, i j k}\right)$, so $i j k=i$. Hence $s a t=\left(s \varphi_{j, i}\right) a\left(t \varphi_{k, i}\right)$. Similarly $s b t=\left(s \varphi_{j, i}\right) b\left(t \varphi_{k, i}\right)$ (for $(s a t, s b t) \in \tau$ implies $\left.s b t \in S_{i}\right)$. Since $s \varphi_{j, i}$ and $t \varphi_{k, i}$ are in $S_{i}$, $(s a t, s b t) \in \sigma$. The result follows from Lemma 2.4 .

THEOREM 5.8. If $S$ is a strong semilatice of semigroups $S_{i}, i \in Y$, with associated congruence $\tau$, then $\Lambda^{\tau}(S)$ is a subdirect product of the lattices $\Lambda\left(S_{i}\right), i \in Y$.

ProOF. This is now immediate from Corollaries 2.2 and 2.3. //

In particular if the congruence classes are all simple then $\tau=\mathscr{J}$ (Petrich (1973), Theorem II.4.5). As noted earlier, little is known about congruence lattices of simple semigroups in general. However by Proposition 4.2 the lattice of congruences on a completely simple semigroup is $M$-symmetric. Now it is known (Petrich (1973), Theorem IV.4.3) that the strong semilattices of completely simple semigroups are precisely the normal bands of groups, that is the unions of groups in which $\mathscr{H}$ is a congruence and $S / \mathscr{H}$ is a normal band. (A band is normal if it satisfies the identity $a b c a=a c b a$.) We have therefore proved

COROLLARY 5.9. If $S$ is a normal band of groups then $\Lambda^{f}(S)$ is $M$-symmetric, whence semimodular. //

This generalizes the theorem of Baird (1972) that $\Lambda^{f}(S)$ is semimodular on a normal band. Using the techniques described in Section 4, and other methods, Eberhart and Williams (1978) claimed (Theorem 2.7) that $\Lambda^{\prime}(S)$ was in fact semimodular on any band of groups. For the non-normal band of Example 5.6 (where the methods of this section fail), however, $\Lambda^{f}(S)$ is not semimodular as we now show.

EXAMPLE 5.10. Let $S$ be the band of Example 5.6. The atoms of $\Lambda^{\mathscr{f}}(S)$ are $\alpha=\{(a, c),(c, a)\} \cup \imath, \beta=\{(b, d),(d, b)\} \cup \imath$ and $\rho=\{(a, b),(c, d),(b, a),(d, c)\} \cup \imath$. Note 
that $\alpha \vee \beta=\alpha \cup \beta \nsubseteq \omega_{K} \cup \imath$ (the Rees congruence modulo $K$ ), but $\omega_{K} \cup \imath>\rho$. Thus $\rho \vee \alpha=\omega_{K} \cup t$, which does not cover $\alpha$. Let $\tau$ be the congruence $\rho \cup\{(s, t),(t, s)\}$. The diagram for $\Lambda^{\mathcal{Y}}(S)$ is given in Figure 1.

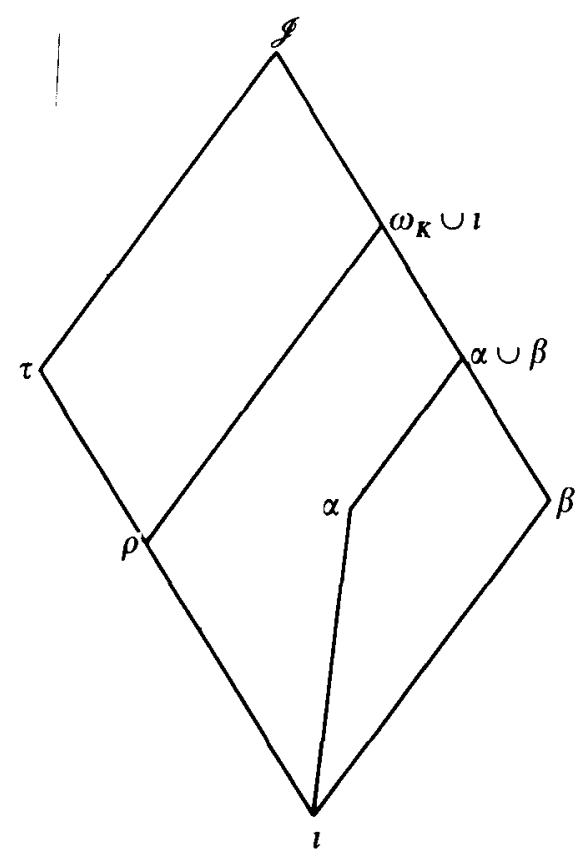

FIGURE 1.

\section{Applications to left congruences}

In this section we consider the left congruences contained in $\mathscr{R}$. Since $\mathscr{R}$ does not in general have principal factors the discussion will be confined to the completely semisimple case. (See Section 3).

If $S$ is any inverse semigroup then $\Lambda_{i}^{\mathscr{R}}(S) \cong \mathscr{L} \mathscr{F}(S)$, the lattice of full inverse subsemigroups of $S$ (Meakin (1975) : an inverse subsemigroup of $S$ is $f$ ull if it contains all the idempotents of $S$ ). The author showed (1978a) that $\mathscr{L} \mathscr{F}(S)$ is semimodular if $S$ is completely semisimple and each $\mathscr{D}$-class either is combinatorial $(\mathscr{H}=i)$ or is itself a group with semimodular subgroup lattice. We will generalize this result to completely semisimple semigroups in general. The first step is the analogue of Lemmas 5.3 and 5.7 .

LEMMA 6.1. Let $S$ be a completely semisimple semigroup and let $R$ be an $\mathscr{R}$-class of $S$. Every 0 -restricted left congruence on the associated principal $\mathscr{R}$-factor is induced by a left congruence on $S$. 
ProOf. Let $\sigma$ be a 0-restricted left congruence on the principal $\mathscr{R}$-factor and suppose $(a, b) \in \sigma, a \neq b$, and $s a \in R$ for some $s \in S^{1}$. Since $\mathscr{R}$ is a left congruence on $S$, $s b \in R$.

Now $S$ is regular, so $a$ has an inverse $a^{\prime}$ in $S$, and $e=a a^{\prime} \mathscr{R} a$ : in fact $e$ is a left identity for $R$. Now ( $s a) a^{\prime}=s e$ and (se) $a=s a$, so $s e \in R$. Hence $s a=(s e) b=s b$. The result now follows from the analogue of Lemma 2.4 for left congruences.

THEOREM 6.2. If $S$ is a completely semisimple semigroup then $\Lambda_{l}^{\#}$ is a subdirect product of the lattices of 0 -restricted left congruences on its principal $\mathbb{R}$-factors.

PROOF. Apply the analogue of Corollary 2.3(ii) for left congruences.

If the $\mathscr{R}$-class $R$ consists of a single $\mathscr{H}$-class, then since $S$ is regular, $R$ is a group, whence the corresponding subdirect factor is just its lattice of subgroups. Now suppose $R$ is combinatorial.

LeMMA 6.3. Let $R$ be a combinatorial $\mathscr{R}$-class of a completely semisimple semigroup $S$. Then every equivalence on $R$ is the restriction of a 0 -restricted left congruence on the associated principal $\mathscr{R}$-factor $P$.

Proof. Let $\alpha$ be an equivalence on $R$ and let $\rho=\alpha \cup\{(0,0)\}$, a 0 -restricted equivalence on $P$. To show $\rho$ is a left congruence on $R$, let $(x, y) \in \rho, x \neq y$ (so that $x, y \in R$ ) and let $r \in R$. If $r x \in R$ then since $P$ is contained in a completely 0 -simple principal ( $\mathscr{J}$ ) factor of $S, r x \in R \cap L_{x}$, whence $L_{r} \cap R$, that is $H_{r}$, contains an idempotent. (See Clifford and Preston (1961), Theorems 2.52 and 2.17 respectively). But since $R$ is combinatorial, $r$ is that idempotent and is therefore a left identity for $R$, so that $(r x, r y)=(x, y) \in \rho$. If $r x=0$ then $r y=0$ similarly. Hence $\rho$ is a left congruence on $P$, inducing $\alpha$.

THEOREM 6.4. If $S$ is completely semisimple semigroup in which each $\mathscr{R}$-class either is combinatorial or is a group with $M$-symmetric lattice of subgroups then $\Lambda_{l}^{2}(S)$ is $M$ symmetric, whence semimodular.

ProOF. The lattice of 0 -restricted congruences on a combinatorial principal $\mathscr{R}$ factor is, by the lemma, isomorphic with the full lattice of equivalences on the corresponding $\mathscr{R}$-class, whence it is $M$-symmetric, by Result 4.1 . Theorem 6.2 may then be applied.

COROLlary 6.5. In any combinatorial completely semisimple semigroup, $\Lambda_{l}^{*}$ is semimodular. In particular $\Lambda_{l}^{*}$ is semimodular in any free inverse semigroup and in any band. // 
In any semigroup, if an $\mathscr{R}$-class is combinatorial so is every $\mathscr{R}$-class in the same $\mathscr{D}$ class. Thus the condition that every $\mathscr{R}$-class be either combinatorial or a group is equivalent to the condition that every $\mathscr{D}$-class be either combinatorial or a left group (that is a direct product of a left zero semigroup and a group), for if $D$ is a noncombinatorial $\mathscr{D}$-class, then each $\mathscr{R}$-class is a group and $D$ consists of a single $\mathscr{L}$ class, whose idempotents form a left zero semigroup. (See Petrich (1973), Theorem IV.3.9.) For inverse semigroups this reduces to the condition stated earlier.

By methods similar to those used in Jones (1978a) it may be shown that the converse to Theorem 6.4 is true, that is $\Lambda_{l}^{*}(S)$ is $M$-symmetric if and only if each $\mathscr{D}$ class of $S$ either is combinatorial or is a left group whose associated group has $M$ symmetric lattice of subgroups. For a full discussion, including the special cases of modularity and distributivity, the reader is referred to the above paper, and for the non-completely semisimple case to Jones (1978b).

Modularity and distributivity of the lattice of left congruences on a band have been studied by Dean and Oehmke (1964).

\section{References}

G. R. Baird (1972), 'On the lattice of congruences on a band', J. Aust. Math. Soc. 14, 49-58.

G. Birkhoff (1967), Lattice theory, 3rd ed. (Amer. Math. Soc., Providence, R.I).

A. H. Clifford and G. B. Preston (1961), Algebraic theory of semigroups, Vol. I, Math. Surveys 7 (Amer. Math. Soc., Providence, R.I.).

A. H. Clifford and G. B. Preston (1967), Algebraic theory of semigroups, Vol. II, Math. Surveys 7 (Amer. Math. Soc., Providence, R.I.).

P. Crawley and R. P. Dilworth (1973), Algebraic theory of lattices (Prentice-Hall, New Jersey).

R. A. Dean and R. H. Oehmke (1964), 'Idempotent semigroups with distributive right congruence lattices', Pacific J. Math. 14, 1187-1209.

C. Eberhart and W. Williams (1978). 'Semimodularity in lattices of congruences', J. Algebra 52, 73-87.

T. E. Hall (1972), 'Congruences and Green's relations on regular semigroups', Glasgow Math. J. 13, $167-175$.

J. M. Howie (1976), An introduction to semigroup theory (Academic Press, London).

J. M. Howie and G. Lallement (1966), 'Certain fundamental congruences on a regular semigroup', Proc. Glascow Math. Assoc. 7, 145-159.

K. G. Johnston (1978), 'Congruence lattices on Rees matrix semigroups', Semigroup Forum 15, 247-262.

P. R. Jones (1978a), 'Semimodular inverse semigroups', J. London Math. Soc. 17, 446456.

P. R. Jones (1978b), 'Distributive inverse semigroups', J. London Math. Soc. 17, 457-466.

G. Lallement (1966), 'Congruences et équivalences de Green sur un demi-groupe régulier', C. R. Acad. Sci. Paris, Sér. A 262, 613-616.

G. Lallement (1967), 'Demi-groupes réguliers', Ann. Mat. Pura Appl. 77, 47-129.

F. Maeda and S. Maeda (1970), Theory of symmetric lattices (Springer, Berlin).

J. Meakin (1975), 'One-sided congruences on inverse semigroups', Trans. Amer. Math. Soc. 206, 67-82.

W. D. Munn (1964), 'A certain sublattice of the lattice of congruences on a regular semigroup', Math. Proc. Cambridge Philos. Soc. 60, 385-391.

M. Petrich (1973), Introduction to semigroups (Merrill Publishing Co., Columbus, Ohio).

H. E. Scheiblich (1970), 'Semimodularity and bisimple $\omega$-semigroups', Proc. Edinburgh Math. Soc. 17, 79-81. 
C. Spitznagel (1973a), 'The lattice of congruences on a band of groups', Glasgow Math. J. 14, 189-197.

C. Spitznagel (1973b), ' $\theta$-modular bands of groups', Trans. Amer. Math. Soc. 177, 469-482.

\section{Mathematics Department}

University of Western Australia

Nedlands, Western Australia 6009

Australia 